Original

\title{
The effect of blood contamination on dislocation resistance of different endodontic reparative materials
}

\author{
Yakup Üstün, Hüseyin S. Topçuoğlu, Firdevs Akpek, and Tuğrul Aslan \\ Department of Endodontics, Faculty of Dentistry, Erciyes University, Kayseri, Turkey
}

(Received January 12, 2015; Accepted April 15, 2015)

\begin{abstract}
This study evaluated the retention characteristics of ProRoot mineral trioxide aggregate (MTA), RetroMTA, Supra MTA, and Biodentine biomaterials used to repair furcation perforations contaminated with blood. Furcal perforations measuring $1.3 \mathrm{~mm}$ in diameter and $2 \mathrm{~mm}$ in height were created in 96 mandibular first molar teeth, which were then randomly divided into the following two groups $(n=$ 48): contaminated $(+)$ or non-contaminated $(-)$ with blood. The groups were subdivided into four groups $(n=12)$ according to the material used (ProRoot MTA, RetroMTA, Supra MTA, and Biodentine) to seal the perforations. The samples were allowed to set for $\mathbf{1 4}$ days and were then subjected to push-out testing. The results were analyzed using ANOVA, and the failure modes were examined using a surgical microscope. ProRoot MTA (+/-) and RetroMTA (+/-) exhibited superior bond strength values; in addition, there were no significant differences among these groups $(P>0.05)$. Biodentine $(+)$ showed intermediate values that were sometimes statistically similar to the ProRoot MTA (+/-) and RetroMTA (+/-) groups (P $>0.05)$ and, at other times, the Biodentine $(-)$ and Supra MTA $(+/-)$ groups $(P>0.05)$. The lowest bond strength values were shown by the Biodentine $(-)$ and Supra MTA groups $(P>0.05)$. "Adhesive failure mode" was the most frequently observed type for all tested materials. Blood contamination did not affect the dislocation resistance of materials.
\end{abstract}

(J Oral Sci 57, 185-190, 2015)

Correspondence to Dr. Yakup Üstün, Endodonti Anabilim Dalı, Erciyes Üniversitesi Diş Hekimliği Fakultesi, 38039 Kayseri, Turkey

E-mail: yakupustun33@hotmail.com

doi.org/10.2334/josnusd.57.185

DN/JST.JSTAGE/josnusd/57.185
Keywords: MTA, Biodentine, dislocation resistance, furcation perforation

\section{Introduction}

Root perforation is one of the most common causes for endodontic failure, and those in the cervical areas of the root and pulp chamber floors have a poorer prognosis than the ones in the middle or apical sections of the root canals. Immediate repair of a cervical perforation is important to avoid loss of bone or epithelial attachment (1-3) and the likely breakdown of periodontal tissues (4).

An ideal perforation repair material should provide adequate seal; exhibit biocompatibility, dimensional stability, insolubility, and radiopacity; and be easy to manipulate and place $(5,6)$. Mineral trioxide aggregate (MTA) is composed of tricalcium silicate, bismuth oxide, dicalcium silicate, small amounts of tricalcium aluminate, tetracalcium aluminoferrite, and calcium sulfate. This cement is currently the material of choice for use in perforation repair because of its superior properties, such as induction of mineralized tissue formation, hydrophilic behavior (7), setting in the presence of blood (8), biocompatibility (9), and antimicrobial activity (10).

MTA has some disadvantages, such as handling difficulties, long setting time, and the risk of inducing tooth discoloration (11-13). Several new brands of MTA products have been introduced into the market in an attempt to overcome these disadvantages.

ProRoot MTA (Dentsply, Tulsa Dental, Tulsa, OK, USA) is one of the most commonly used brands, and it is used to form apical barriers in immature apices, root-end fillings, direct pulp cappings, pulpotomies and in root perforation repairs $(5,8)$.

A new type of MTA (RetroMTA; BioMTA, Seoul, Republic of Korea) has recently been introduced 
for use as an endodontic reparative material. The manufacturer claims that it contains calcium zirconia complex as a radiopacifier and that it is biocompatible. They also report a shorter setting time in comparison with MTA and the absence of discoloration, even with blood contamination. Chung et al. (14) investigated the effects of ProRoot MTA, Endocem Zr, and RetroMTA cements on cell viability and angiogenic factor release in human pulp-derived cells. They reported that the effect of RetroMTA on human pulp cells is similar in terms of biocompatibility and angiogenic effects to ProRoot MTA. Therefore, it can potentially be as effective in pulp cappings as ProRoot MTA.

Supra MTA (Sarp Dental Ltd, Istanbul, Turkey) is a new tricalcium silicate-based endodontic reparative material. The manufacturer claims that it contains hydrophilic particles of mineral oxides and tricalcium silicate and that it is biocompatible, dimensionally stable, insoluble, and radiopaque. Additionally, this material has a short setting time, does not induce tooth discoloration, and forms a waterproof gel-like structure when mixed with water.

Biodentine (Septodont, St-Maur-des-fosses Cedex, France) was introduced as an alternative to MTA. The powder is mainly composed of tricalcium silicate cement, with a small portion of dicalcium silicate, calcium carbonate, and zirconium oxide $(11,12,15)$. The liquid used to mix the cement consists of calcium chloride (as an accelerator to reduce setting time) and a hydrosoluble polymer. This material is indicated for pulp cappings, retrograde fillings, perforation repairs, apexifications, and temporary fillings $(12,16,17)$.

Perforations that occur during root canal treatments should be immediately sealed using a biocompatible material resistant to dislodging forces applied during restorative procedures and functional activities. Therefore, it is important to use a push-out assay to evaluate the dentin bond strength of reparative materials. Although several studies have assessed the bond strength of MTA and other materials to dentin $(2,3,18)$, none of them have compared the dislocation resistance of new MTA products (Supra MTA and RetroMTA) and Biodentine. Hence, the aim of the present study was to evaluate the effect of blood contamination on the dislocation resistance of different endodontic materials used to repair perforations in the furcal area.

\section{Materials and Methods}

This study used 96 human mandibular first molars with mature apices, and no signs of any carious lesions, shape or size anomalies, fused roots, or previous root canal treatments. Ethical approval was obtained from the review committee of the research foundation of Erciyes University of Medical Sciences in Kayseri, Turkey (2015/121), prior to investigation.

The teeth were cleaned, so as to remove any soft tissue remnants, and stored in $0.5 \%$ chloramine-T solution until use. They were decoronated at the cementoenamel junction using a low-speed diamond-coated saw (Minitom, Struer, Denmark) under water cooling and were then mounted in acrylic molds, leaving a space of $3 \mathrm{~mm}$ under the furcal area for further placement of Gelatamp (RoekoColtène/Whaledent, Langenau, Germany), which acted as a matrix for packing materials during furcal perforation repair (Fig. 1). Perforations were created using \#1.3 cylindrical bur (1.3 $\mathrm{mm}$ diameter), perpendicular to the furcal floor and parallel to the tooth axis (Fig. 2). A periodontal probe was used to measure penetration depth ( $2 \mathrm{~mm}$ ). Samples less than $2 \mathrm{~mm}$ in depth were excluded, while those with greater depth were ground using a diamond disk, to remove excess dentin at the furcal perforation. All the samples were rinsed with distilled water to remove debris produced during the procedure.

Before sealing the perforations with the tested materials, the cavities were assigned to two groups based on contamination $(+)$ or non-contamination $(-)$ with blood $(n=48)$.

To simulate contamination, a 27-gauge syringe was used to inject the perforation cavities with blood, provided by one of the researchers. As this blood was collected immediately before the procedures, no anticoagulant substance was necessary. A piece of Gelatamp was packed under the furcal area, and excess blood was removed with paper points (size 40, taper 0.02), without touching the walls of the perforated dentin (Fig. 2).

Each group was then subdivided into four categories ( $n$ $=12$ ), based on the material used to repair the perforation. The subgroups were as follows: Biodentine, RetroMTA, ProRoot MTA, and Supra MTA.

RetroMTA and ProRoot MTA were mixed according to the manufacturers' instructions (powder/liquid ratio). Cements dispensed in capsules, such as Biodentine and Supra MTA, were mixed for $30 \mathrm{~s}$ using an amalgam mixer.

Four experienced endodontists filled the contaminated $(+)$ and uncontaminated $(-)$ perforations with the tested materials using condensers (1M Markley de amalgam plugger, Hu Friedy, Chicago, IL, USA). Cotton pellets, wet with normal saline, were placed over the reparative material in each tooth. All samples were kept in an incubator at $37^{\circ} \mathrm{C}$ and $95 \%$ relative humidity for 14 days, after which they were subjected to a push-out test. 


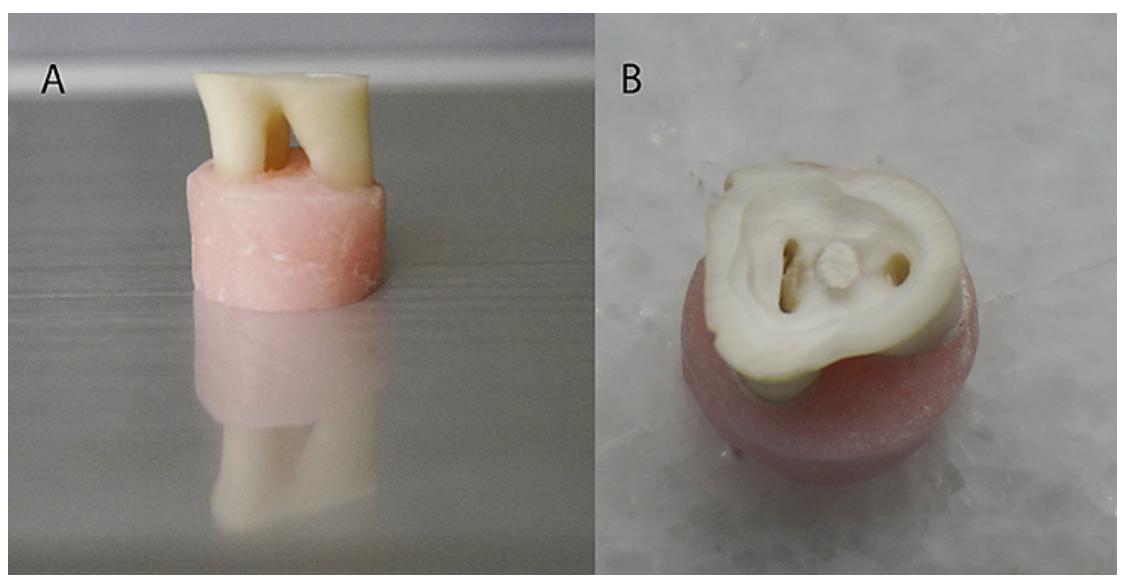

Fig. 1 A: Teeth mounted in acrylic resin, B: Occlusal view of a repaired furcal perforation.

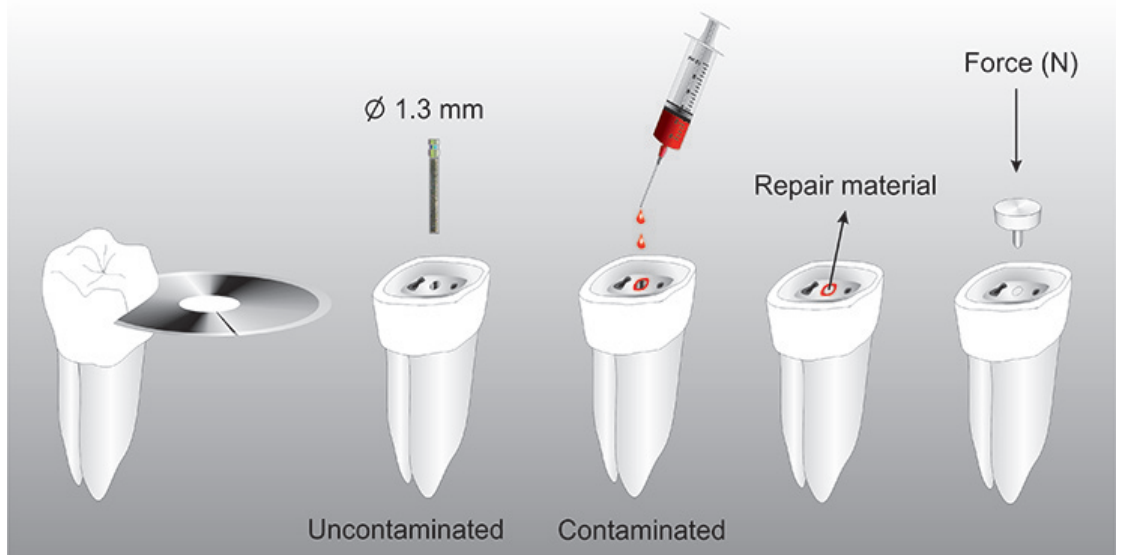

Fig. 2 Application steps of the experimental study.

\section{Push-out test}

The push-out test was conducted using a universal test machine (Instron Corp., Canton, MA, USA). The material placed in the perforation cavity was subjected to a load at a crosshead speed of $1 \mathrm{~mm} / \mathrm{min}$ in the apical direction and parallel to the long axis of the tooth, using a cylindrical plunger with $1.0 \mathrm{~mm}$ diameter, until dislodgement occurred. The maximum load applied to dislodge the reparative material was recorded in Newtons.

The push-out bond strength was calculated in $\mathrm{MPa}$ using this formula:

(Force needed to dislodge the material) / ( $\pi \times$ diameter of perforation site $\times$ height of perforation)

\section{Failure mode analysis}

After the push-out test, the samples were split in half and examined with a surgical microscope (OPMI Pico, Carl Zeiss, Oberkochen, Germany) at $\times 10$ magnification to evaluate failure modes (18): adhesive (failure at the dentin-material interface), cohesive (failure within the material), mixed failure, or a combination of adhesive and cohesive failures.

\section{Statistical analysis}

Statistical analyses were performed with SPSS 13.0 (SPSS for Windows; SPSS Inc., Chicago, IL, USA). The distribution of the data was assessed using the ShapiroWilk normality test and Levene's variance homogeneity test, and one way ANOVA and post hoc Tamhane T2 tests were used for analysis.

\section{Results}

Mean, standard deviations, and percentages of failure patterns of the experimental groups are listed in Table 1.

There were no statistically significant differences between contaminated and uncontaminated groups of the same tested material $(P>0.05)$.

ProRoot MTA (+/-) and RetroMTA (+/-) exhibited superior bond strength values; in addition, there were no significant differences among these groups $(P>$ 
Table 1 The mean, standard deviation, and percentages of failure mode analysis of each test material

\begin{tabular}{lccc}
\hline Groups & Mean $(\mathrm{MPa})$ & Standard deviation $(\mathrm{MPa})$ & Failure mode $\%(\mathrm{~A}, \mathrm{C}, \mathrm{M})$ \\
\hline Biodentine $(-)$ & $3.58^{\mathrm{b}}$ & 1.49 & $75,17,8$ \\
Biodentine $(+)$ & $4.36^{\mathrm{ab}}$ & 2.55 & $90,10,0$ \\
RetroMTA $(-)$ & $7.57^{\mathrm{a}}$ & 3.5 & $66,17,17$ \\
RetroMTA (+) & $6.37^{\mathrm{a}}$ & 0.82 & $50,30,20$ \\
ProRoot MTA $(-)$ & $7.38^{\mathrm{a}}$ & 4.17 & $55,8,36$ \\
ProRoot MTA $(+)$ & $8.34^{\mathrm{a}}$ & 3.9 & $75,17,8$ \\
Supra MTA $(-)$ & $2.83^{\mathrm{b}}$ & 1.94 & $75,17,8$ \\
Supra MTA $(+)$ & $2.32^{\mathrm{b}}$ & 1.01 & $84,8,8$ \\
\hline
\end{tabular}

A, adhesive; C, cohesive; M, mixed; MTA, mineral trioxide aggregate. Common superscript letters do not show any significant difference.

0.05). The contaminated group of Biodentine $(+)$ showed intermediate values, which were sometimes statistically similar to the ProRoot MTA (+/-) and Retro MTA (+/-) groups $(P>0.05)$, and at other times similar to the uncontaminated Biodentine (-) and Supra MTA $(+/-)$ groups $(P>0.05)$. The lowest bond strength values were shown by the Biodentine (-) and Supra MTA groups $(P$ $>0.05)$.

Moreover, failure mode analysis showed that the tested materials mainly exhibited adhesive failures.

\section{Discussion}

Previous studies reported that the setting reaction of MTA products may continue for a month in the presence of moisture and phosphate-buffered saline solutions (19). This means that the time elapsed after placement of the material affects its retention characteristics (2,20-22). Vanderweele et al. (2) reported that a greater dislodgement resistance is observed when MTA is allowed to set for 7 days, as compared with setting times of only 24-72 h. According to these authors, MTA should be left undisturbed for 7 days before placement of a coronal restoration to decrease the risk of displacement. Therefore, in the present study, reparative materials were stored at $95 \%$ relative humidity for 2 weeks to ensure a complete setting process.

The present study was designed to test the dislodgement resistance of different furcation reparative materials, with or without blood contamination. In contaminated samples, a hemorrhagic situation was simulated at the furcal area by filling the perforations with blood and removing the excess using paper points.

Our results showed that all MTA products had better dislodgement resistance than Biodentine, with the exception of Supra MTA. There was no significant difference between the contaminated and uncontaminated subgroups of the same tested material. A previous study reported that MTA shows a calcium phosphate phase transformation in the presence of phosphatecontaining mediums (23). It has also been reported that when phosphate-containing solutions are used as storage medium, the push-out bond strength of MTA increases (24). This can be explained by the ability of MTA to form hydroxyapatite in the presence of phosphate-containing fluids. In the present study, although the contaminated groups showed higher retention values than the uncontaminated ones, intragroup comparisons showed no statistically significant differences. This could be due to an insufficient period of storage (14 days), which may not have allowed crystal deposition. This would also explain the similarity observed between the contaminated and non-contaminated groups. This result contradicts the study by Rahimi et al. (3) who investigated the effects of blood contamination on the dislodgement resistance of endodontic repair materials MTA (Dentsply, Tulsa Dental) and calcium-enriched mixture (CEM) and reported that contamination with blood had a deleterious effect on the bond strength of biomaterials.

When a tricalcium silicate-based material was placed in the root canal, hydroxyapatite crystals nucleated, grew, and filled the spaces between the material and the dentin walls. Although mechanical sealing occurs initially, it is thought that with time a diffusion-controlled reaction between the apatite layer and dentin leads to chemical bonding by the phosphate-buffered solutions, thus creating a seal at the MTA-dentine interface (25). Hydroxyapatite crystals deposit along the collagen fibers at this material-dentin interface and form the tag-like structures that are responsible for mechanical bonding $(21,26)$. The phosphate for the reparative materials is 
supplied by blood, and the formation of mechanical bonding results in higher retention values in the contaminated subgroups. However, the results of the present study do not support this hypothesis as this was likely limited by the short period of sample storage (14 days).

Kim et al. (27) investigated the formation of the interfacial layer (Biodentine-dentin and MTA-dentin) and found that the thickness is much greater with MTA $(14.5 \mu \mathrm{m})$ than with Biodentine $(4.8 \mu \mathrm{m})$. They attributed this finding to the shorter setting time of Biodentine, which likely resulted in a reduced amount of time for interaction between the calcium from Biodentine and the phosphate from simulated body fluid (SBF). The authors also observed multiple gaps in the dentin-Biodentine interface caused by excessive moisture. According to the manufacturer's instructions, Biodentine should not be exposed to water or any other fluids during the first hours of the setting reaction. However, in the present study, materials were kept in 95\% relative humidity using wet cotton pellets until the push-out test was performed. This excessive moisture may have adversely affected the setting reaction of Biodentine and resulted in separation from the dentin. This would explain the inferior bond strength values observed in the Biodentine group. Moreover, Supra MTA has a very short working time $(2 \mathrm{~min})$ and an average setting period $(2 \mathrm{~h})$. This explains the low bond strength values observed with this material because the short storage and setting time may have precluded formation of the interfacial layer between dentin and reparative material.

Contrary to our results, Guneser et al. (11) reported that Biodentine shows greater push-out strength than MTA. However, the effects of blood contamination were not investigated in this study, and the differences in the results can be explained by the different methodologies used.

In the present study, adhesive bond failure was the most frequent pattern of failure observed. These findings are similar to the study performed by Vanderweele et al. (2), who reported that MTA dentin bond failures were usually of the adhesive type. Sarkar et al. (25) showed that teeth filled with MTA and stored in synthetic tissue fluid for 2 months produced an adherent interfacial layer at the dentin wall. They stated that this interfacial layer allows formation of a chemical bond between the MTA and the dentinal walls. In this study, the adhesive type of bond failure may be attributed to the short duration of sample storage before push-out testing.

Coronal restoration of teeth with repaired furcal perforations is very important for the success of the treatment. Hashemet et al. (28) stated that the pressure applied during the condensation of amalgam was 9.2 and 5.5 MPa for small and medium-sized condensers, respectively. Thus, the results of the present study indicate that ProRoot MTA and RetroMTA seem to be more suitable for use as a reparative material in case of furcal perforations.

Further studies are needed to investigate the influence of different setting periods and blood contamination on the dislodgment resistance of tricalcium silicate-based endodontic materials.

\section{Acknowledgments}

The authors would like to thank Dr. Shin Yoojin and the manufacturer (BioMTA, Seoul, Republic of Korea) for their support in RetroMTA.

\section{References}

1. Jew RC, Weine FS, Keene JJ Jr, Smulson MH (1982) A histologic evaluation of periodontal tissues adjacent to root perforations filled with Cavit. Oral Surg Oral Med Oral Pathol 54, 124-135.

2. Vanderweele RA, Schwartz SA, Beeson TJ (2006) Effect of blood contamination on retention characteristics of MTA when mixed with different liquids. J Endod 32, 421-424.

3. Rahimi S, Ghasemi N, Shahi S, Lotfi M, Froughreyhani M, Milani AS et al. (2013) Effect of blood contamination on the retention characteristics of two endodontic biomaterials in simulated furcation perforations. J Endod 39, 697-700.

4. Setzer FC, Boyer KR, Jeppson JR, Karabucak B, Kim S (2011) Long-term prognosis of endodontically treated teeth: a retrospective analysis of preoperative factors in molars. $\mathrm{J}$ Endod 37, 21-25.

5. Ford TR, Torabinejad M, McKendry DJ, Hong CU, Kariyawasam SP (1995) Use of mineral trioxide aggregate for repair of furcal perforations. Oral Surg Oral Med Oral Pathol Oral Radiol Endod 79, 756-763.

6. Fuss Z, Trope M (1996) Root perforations: classification and treatment choices based on prognostic factors. Endod Dent Traumatol 12, 255-264.

7. Tomson PL, Grover LM, Lumley PJ, Sloan AJ, Smith AJ, Cooper PR (2007) Dissolution of bio-active dentine matrix components by mineral trioxide aggregate. J Dent 35, 636-642.

8. Parirokh M, Torabinejad M (2010) Mineral trioxide aggregate: a comprehensive literature review--Part I: chemical, physical, and antibacterial properties. J Endod 36, 16-27.

9. Ribeiro DA, Matsumoto MA, Duarte MA, Marques ME, Salvadori DM (2005) In vitro biocompatibility tests of two commercial types of mineral trioxide aggregate. Braz Oral Res 19, 183-187.

10. Lovato KF, Sedgley CM (2011) Antibacterial activity of endosequence root repair material and proroot MTA against clinical isolates of Enterococcus faecalis. J Endod 37, 15421546 . 
11. Guneser MB, Akbulut MB, Eldeniz AU (2013) Effect of various endodontic irrigants on the push-out bond strength of biodentine and conventional root perforation repair materials. J Endod 39, 380-384.

12. Zhou HM, Shen Y, Wang ZJ, Li L, Zheng YF, Häkkinen L et al. (2013) In vitro cytotoxicity evaluation of a novel root repair material. J Endod 39, 478-483.

13. Marciano MA, Costa RM, Camilleri J, Mondelli RF, Guimarães BM, Duarte MA (2014) Assessment of color stability of white mineral trioxide aggregate angelus and bismuth oxide in contact with tooth structure. J Endod 40, 1235-1240.

14. Chung CJ, Kim E, Song M, Park JW, Shin SJ (2015) Effects of two fast-setting calcium-silicate cements on cell viability and angiogenic factor release in human pulp-derived cells. Odontology, doi: 10.1007/s10266-015-0194-5.

15. Han L, Okiji T (2011) Uptake of calcium and silicon released from calcium silicate-based endodontic materials into root canal dentine. Int Endod J 44, 1081-1087.

16. Koubi S, Elmerini H, Koubi G, Tassery H, Camps J (2012) Quantitative evaluation by glucose diffusion of microleakage in aged calcium silicate-based open-sandwich restorations. Int J Dent, 105863.

17. Koubi G, Colon P, Franquin JC, Hartmann A, Richard G, Faure MO et al. (2013) Clinical evaluation of the performance and safety of a new dentine substitute, Biodentine, in the restoration of posterior teeth--a prospective study. Clin Oral Investig 17, 243-249.

18. Shokouhinejad N, Nekoofar MH, Iravani A, Kharrazifard MJ, Dummer PM (2010) Effect of acidic environment on the push-out bond strength of mineral trioxide aggregate. J Endod 36, 871-874.

19. Gancedo-Caravia L, Garcia-Barbero E (2006) Influence of humidity and setting time on the push-out strength of mineral trioxide aggregate obturations. J Endod 32, 894-896.
20. Sluyk SR, Moon PC, Hartwell GR (1998) Evaluation of setting properties and retention characteristics of mineral trioxide aggregate when used as a furcation perforation repair material. J Endod 24, 768-771.

21. Reyes-Carmona JF, Felippe MS, Felippe WT (2009) Biomineralization ability and interaction of mineral trioxide aggregate and white portland cement with dentin in a phosphate-containing fluid. J Endod 35, 731-736.

22. Chedella SC, Berzins DW (2010) A differential scanning calorimetry study of the setting reaction of MTA. Int Endod J 43, 509-518.

23. Tay FR, Pashley DH, Rueggeberg FA, Loushine RJ, Weller RN (2007) Calcium phosphate phase transformation produced by the interaction of the Portland cement component of white mineral trioxide aggregate with a phosphate-containing fluid. J Endod 33, 1347-1351.

24. de Almeida J, Felippe MC, Bortoluzzi EA, Teixeira CS, Felippe WT (2014) Influence of the exposure of MTA with and without calcium chloride to phosphate-buffered saline on the push-out bond strength to dentine. Int Endod J 47, 449-453.

25. Sarkar NK, Caicedo R, Ritwik P, Moiseyeva R, Kawashima I (2005) Physicochemical basis of the biologic properties of mineral trioxide aggregate. J Endod 31, 97-100.

26. Reyes-Carmona JF, Felippe MS, Felippe WT (2010) A phosphate-buffered saline intracanal dressing improves the biomineralization ability of mineral trioxide aggregate apical plugs. J Endod 36, 1648-1652.

27. Kim JR, Nosrat A, Fouad AF (2015) Interfacial characteristics of Biodentine and MTA with dentine in simulated body fluid. J Dent 43, 241-247.

28. Hashem AA, Wanees Amin SA (2012) The effect of acidity on dislodgment resistance of mineral trioxide aggregate and bioaggregate in furcation perforations: an in vitro comparative study. J Endod 38, 245-249. 